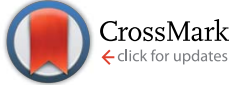

Cite this: RSC Adv., 2017, 7, 1032

Received 14th October 2016 Accepted 21st November 2016

DOI: $10.1039 / c 6 r a 25236 b$

www.rsc.org/advances

\section{Effects of Osteo-F, a new herbal formula, on osteoporosis via up-regulation of Runx2 and Osterix}

\author{
Ji Eun Lee, $\dagger^{\mathrm{a}}$ Mi Hye Kim, $\uparrow^{\mathrm{a}}$ Jongki Hong, ${ }^{\mathrm{b}}$ Hyuck Jai Choi, ${ }^{\mathrm{c}}$ Jongrak Park ${ }^{\mathrm{d}}$ \\ and Woong Mo Yang*a
}

Osteoporosis is characterized by low bone mass and structural weakness, resulting in a high risk of fracture. The aim of this study is to investigate the ameliorative effects of a herbal formula, Osteo-F, containing Schizandra chinensis, Lycium chinensis and Eucommia ulmoides, on osteoporosis. Female ICR mice were randomly assigned to a sham-operated group (Sham) and five ovariectomized (OVX) groups: OVX with vehicle (OVX), OVX with calcium (Ca), and OVX with 1, 10 and $100 \mathrm{mg}$ per kg per day Osteo-F (OF1, OF10 and OF100). Oral administration of Ca or Osteo-F started 7 weeks after OVX and lasted for 13 weeks. The femurs were collected to analyze the bone mineral content (BMC) and bone mineral density (BMD) and bone histology. Blood was collected to examine serum calcium concentration. In addition, the expressions of Runx2 and Osterix in SaOS-2 osteoblast cells were analyzed to confirm the mechanism on osteoblast differentiation. In the present study, Osteo-F treatment significantly restored the low BMC and BMD in the femur. As shown in histological analysis, hyperplasia of the growth plate in the epiphyseal plate was markedly recovered in Osteo-F-treated bone. In addition, serum calcium concentrations were increased with Osteo-F treatment. In the in vitro study, the expressions of Runx2 and Osterix were significantly increased in Osteo-F-treated SaOS-2 osteoblast cells. These results indicate that oral administration of Osteo-F, a newly developed formula, has ameliorative effects on osteoporosis by increasing osteoblast related markers, such as Runx2 and Osterix.

\section{Introduction}

Since bone is a major tissue that provides internal skeletal support, debilitating bone diseases, including osteoporosis, osteopetrosis, osteogenesis imperfecta and Paget's disease, are serious dangers during the remaining life time. ${ }^{1}$ In particular, over 200 million people worldwide suffer from osteoporosis, one of the common skeletal disorders., ${ }^{2,3}$ Osteoporosis is characterized by low bone mass and bone mineral density (BMD), resulting in an increase of fracture risk. ${ }^{4}$

Although various reasons could induce osteoporosis, estrogen deficiency is known to disrupt the equilibrium of bone remodeling. ${ }^{2}$ A number of recent studies have demonstrated that estrogen plays roles as a regulator of bone turnover and population of osteoclasts. ${ }^{5}$ Therefore, osteoporosis is highly distributed in post-menopausal women who have stopped

\footnotetext{
${ }^{a}$ Department of Convergence Korean Medical Science, Graduate School, Kyung Hee University, Seoul 02447, Republic of Korea. E-mail: wmyang@khu.ac.kr; Fax: +82-2961-2209; Tel: +82-2-961-2209

${ }^{b}$ College of Pharmacy, Kyung Hee University, Seoul, Republic of Korea

${ }^{c}$ East-West Medical Research Institute, Kyung Hee University, Seoul, Republic of Korea

${ }^{d}$ Mungyeong Omijavalley Inc., Mungyeong, Republic of Korea

$\uparrow$ These authors contributed equally.
}

having ability to produce estrogen. ${ }^{6}$ For that reason, hormone replacement therapy (HRT) is primarily used to treat postmenopausal osteoporosis. However, increase of cancer risk by long-term use of HRT is concern in osteoporotic patients. ${ }^{7}$ Prescribed bisphosphonate to prevent bone loss in postmenopausal women is reported to induce atrial fibrillation and osteonecrosis of the jaw. ${ }^{\mathbf{8} 9}$ Thus approach to find alternative treatment of osteoporosis with no adverse effects is desirable.

Osteo-F is a newly developed herbal formula consisted of the fruit of Schizandra chinensis, Lycium chinensis and radix of Eucommia ulmoides based on the classic book. According to the Korean traditional literature, S. chinensis, L. chinensis and $E$. ulmoides have been used for treating bone disease. ${ }^{10}$ Additionally, studies regarding on their pharmacological relevancies were recently published. In previous study, S. chinensis exhibited ameliorative effect on osteoporosis in ovariectomized (OVX) mice by increasing estrogen receptor- $\alpha$ and $-\beta .{ }^{\mathbf{1 1}}$ L. chinensis exerted an inhibition of osteoclast formation. ${ }^{12}$ The bone remodeling-related factors, Osterix, runt-related transcription factor 2 (Runx2), receptor activator of nuclear factor kappa-B ligand (RANKL) and osteoprotegerin (OPG) were regulated by E. ulmoides in SaOS-2 osteoblast cells. ${ }^{13}$

Based on previous evidences, we combined a new formula to improve osteoporosis. In this study, the effects of Osteo-F on 
osteoporosis were determined to analyze the bone mineral content (BMC) and BMD in OVX mice. To understand its mechanism of actions on bone remodeling, the expressions of Runx2 and Osterix in SaOS-2 cells were further investigated.

\section{Experimental}

\subsection{Preparation of Osteo-F}

The dried fruit of $S$. chinensis and $L$. chinensis, and the radix of $E$. ulmoides were obtained from Jung-do Herb (Seoul, Korea). Each $200 \mathrm{~g}$ of $S$. chinensis and L. chinensis, and E. ulmoides were extracted twice with $2 \mathrm{~L}$ of distilled water, respectively, for $2 \mathrm{~h}$ under reflux at $100{ }^{\circ} \mathrm{C}$. Then the extracts were evaporated using BÜCHI Rotavapor R-220 (BÜCHI Labortechnik, Switzerland) and freeze-dried using lyophilizer (Ilshin Biobase, Korea). The final yields of each herb were $35 \%, 31.8 \%$ and $10.1 \%$, respectively. Each voucher specimens (SC-W100, LC-W100 and EUW100) were deposited at our laboratory. Osteo-F consists of $S$. chinensis, L. chinensis and E. ulmoides according to the dilution ratio $2: 1: 1$, respectively. For the treatment, $60 \mathrm{mg} S$. chinensis, $30 \mathrm{mg}$ L. chinensis and $10 \mathrm{mg}$ E. ulmoides extract powders were mixed and dissolved in $1 \mathrm{~mL}$ distilled water (finally prepared $100 \mathrm{mg} \mathrm{mL}{ }^{-1}$ Osteo-F), considered on the final yields of each extract.
The quality evaluation of $S$. chinensis, L. chinensis and $E$. ulmoides was performed with three reference compounds of schizandrin, betaine and geniposidic acid, respectively, by highperformance liquid chromatography diode array detector (HPLC-DAD) on an Agilent Series 1100 HPLC system. Chromatographic separation was achieved using the Atlantis HILIC silica $(4.6 \times 150 \mathrm{~mm}, 5 \mu \mathrm{m})$. The eluent for schizandrin, betaine and geniposidic acid consisted of $30 \mathrm{mM}$ ammonium acetate and acetonitrile at a flow rate of $1.0 \mathrm{~mL} \mathrm{~min}^{-1}$. The detection wavelength was $220 \mathrm{~nm}$ at $30{ }^{\circ} \mathrm{C}$. The peaks of schizandrin, betaine and geniposidic acid were synchronized with their standards (Fig. 1). The concentrations of schizandrin, betaine and geniposidic acid were $273.03 \mu \mathrm{g} \mathrm{mL} \mathrm{mL}^{-1}(1.82 \%), 360.32 \mu \mathrm{g}$ $\mathrm{mL}^{-1}(1.44 \%)$ and $19.56 \mu \mathrm{g} \mathrm{mL^{-1 }}(0.07 \%)$, respectively.

\subsection{Mice}

ICR female mice aged 6 weeks were purchased from RAONBIO Inc. (Yongin, Korea). Mice were maintained in an air-conditioned room and a $12 \mathrm{~h}$ light-dark cycle with freely available of food and water. All experiments were conducted according to the guidelines of the Guide for the Care and Use of Laboratory Animals of the National Institutes of Health. The protocol was approved by
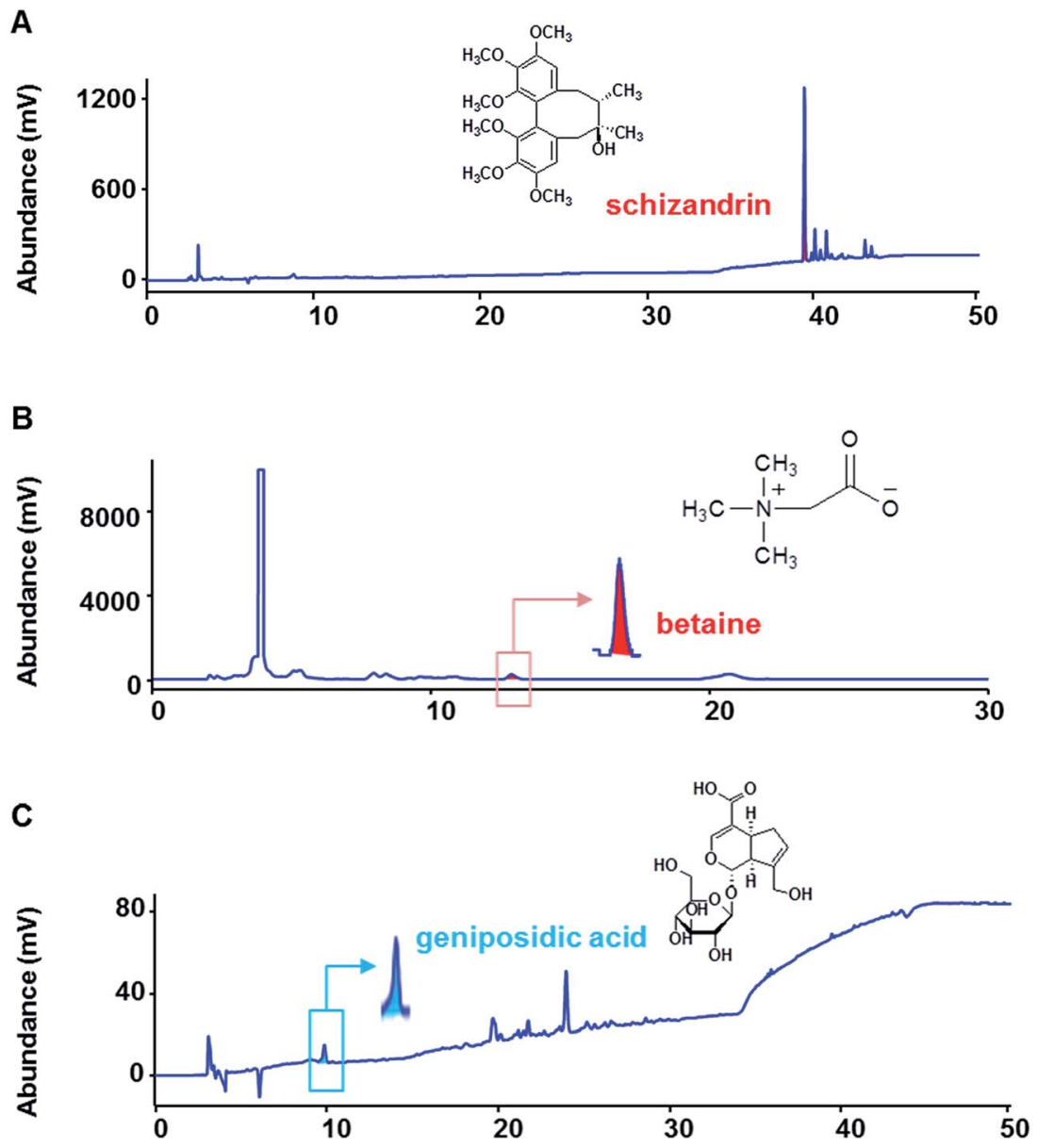

Fig. 1 High-performance liquid chromatography (HPLC) analysis of Schizandra chinensis, Lycium chinensis and Eucommia ulmoides. 
Committee on Care and Use of Laboratory Animals of the Kyung Hee University (KHUASP(SE)-15-093).

\subsection{Induction of ovariectomy-induced osteoporosis}

After 1 week of adaption, the mice were randomly divided into six groups $(n=7)$; Sham, OVX, Ca (calcium chloride-treated group), OF1, OF10 and OF100 (Osteo-F-treated groups at the doses of 1, 10 and $100 \mathrm{mg}$ per kg per day, respectively). All mice except Sham group were OVX and seven mice in Sham group were sham-operated. To recover and induce osteoporosis, mice were maintained for 7 weeks. After that, Sham and OVX groups were treated with the vehicle (PBS containing 1\% DMSO). $150 \mathrm{mg} \mathrm{kg}^{-1}$ of calcium chloride (Sigma, MO, USA) was orally administrated to Ca group as positive control. And Osteo-F was orally treated five times per week during 6 weeks. The mice were sacrificed after blood collection by cardiac puncture. The both side of femurs were obtained to analyze the BMC, BMD and bone histology.

\subsection{Bone histology}

The right femurs were fixed in $10 \%$ neutralized formalin for $18 \mathrm{~h}$ and demineralized with $0.1 \mathrm{M}$ ethylene diamine tetraacetic acid aqueous solution for 1 month. After demineralization, femur samples were dehydrated by ethanol and xylene. Paraffinembedded femur samples were sagittal sectioned into glass slides. The slides were stained with hematoxylin and eosin. Histological changes were monitored under a light microscope using the Leica Application Suite (LAS; Leica Microsystems, Buffalo Grove, IL, USA). Digital images were taken at a magnification of $\times 40$.

\subsection{Measurement of BMC and BMD}

The left femurs were stored in 10\% neutralized formalin until use. The levels of BMC and BMD of the left femurs were determined by dual-energy X-ray absorptiometry with an InAlyzer instrument (MEDIKORS, Seoul, Korea).

\subsection{Serum analysis}

Collected blood samples were centrifuged at $14000 \mathrm{rpm}$ for $30 \mathrm{~min}$ to obtain serum. The serum levels of calcium and magnesium were estimated by using commercial ELISA kit (Adipogen, Shizuoka, Japan) according to the manufacturers' instructions. The optical density was read at $570 \mathrm{~nm}$ and $660 \mathrm{~nm}$ using a microplate reading instrument (BioTek, PA, USA).

\subsection{Cell culture and proliferation analysis}

SaOS-2 cells (human osteoblastic cell line) were maintained in Dulbecco's modified minimal essential medium (DMEM; Gibco, Grand Island, NY, USA) supplemented with 1\% penicillin and 10\% heat-inactivated fetal bovine serum (FBS) at $37{ }^{\circ} \mathrm{C}$ in an atmosphere containing $5 \% \mathrm{CO}_{2}$ that $95 \%$ humidity. The culture medium was changed every 3-4 day.

The cells were seeded into 96-well plates at a density of $1 \times$ $10^{6}$ cells per well. After $24 \mathrm{~h}$, the cells were treated with culture medium containing different concentrations of Osteo-F extract $\left(1,10\right.$ and $\left.100 \mu \mathrm{g} \mathrm{mL}^{-1}\right)$ for $24 \mathrm{~h}$. Then, 3-(4,5-dimethylthiazol-2yl)-2,5-diphenyltetrazolium (MTT) solution $\left(2 \mathrm{mg} \mathrm{mL}^{-1}\right)$ was added into each well and cultured for $2 \mathrm{~h}$ at $37^{\circ} \mathrm{C}$. Afterwards, MTT medium was discarded and DMSO was added. The absorbance at $570 \mathrm{~nm}$ was measured. The experiments were performed in triplicate.

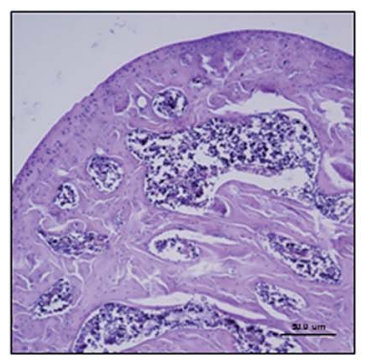

Sham

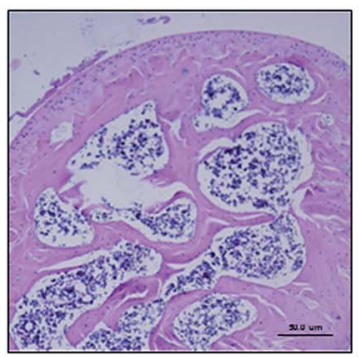

OF1

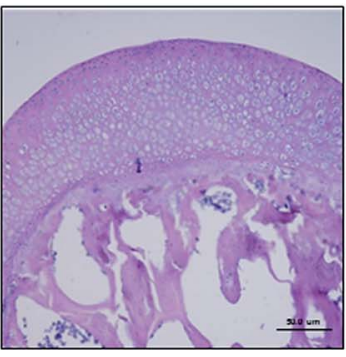

OVX

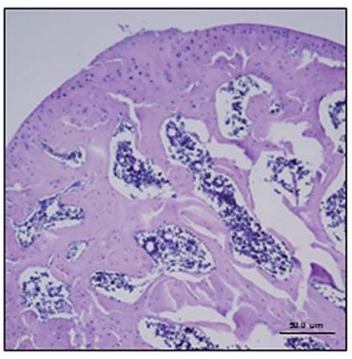

OF10

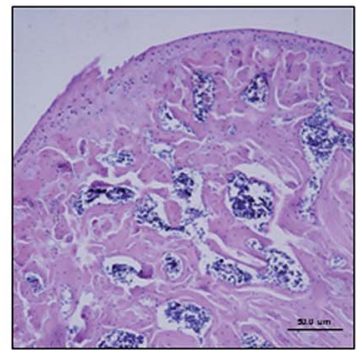

$\mathrm{Ca}$

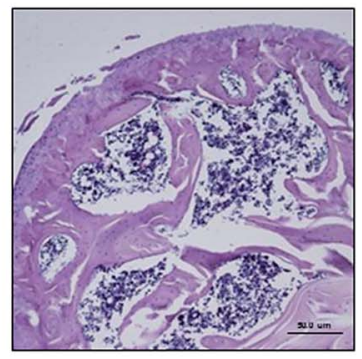

OF100

Fig. 2 The effects of Osteo-F on the growth plate thickness of femur. The sagittal sections were stained with hematoxylin and eosin ( $\mathrm{H} \& \mathrm{E})$ and the magnification was $\times 200$. 


\subsection{Western blotting analysis}

RIPA buffer (50 mM Tris- $\mathrm{HCl}$ (pH 7.4), 1\% Nonidet P-40, 0.5\% sodium deoxycholate, $150 \mathrm{mM} \mathrm{NaCl}$ ) containing protease inhibitors (Roche, Hoffmann, USA) was used for cell protein extraction. The protein concentration was determined using the Bradford protein assay. $20 \mu \mathrm{g}$ of protein was denatured with sodium dodecylsulfate buffer, then electrotransferred onto a PVDF membrane (Bio-Rad, Hercules, CA, USA). Primary antibody (diluted 1 : 1000 in 3\% bovine serum albumin; Cell Signaling, USA) was then added to the membrane. Secondary antibodies (Santa Cruz, CA, USA) were incubated for $2 \mathrm{~h}$ at RT. The membranes were then visualized using an enhanced chemiluminescence detection reagent (Amersham Pharmacia, Piscataway, NJ, USA).

\subsection{Statistical analysis}

Significance was determined by one-way analysis of variance (ANOVA) and Dunnett's multiple comparison tests. In all analyses, $p<0.05$ was taken to indicate statistical significance.

\section{Results and discussion}

The maintenance of bone homeostasis is controlled by constant replenishment as known as bone remodeling. ${ }^{14}$ The two equal, opposite and balanced forces of bone formation by osteoblasts and bone resorption by osteoclasts regulate bone equilibrium. ${ }^{15}$ Interaction of bone-forming cells and bone-resorbing cells occurs on the bone surface during growth of bone. ${ }^{16}$ For that reason, imbalance of bone metabolism leads to disruption of bone remodeling process. ${ }^{17}$ Predominant bone resorption by osteoclast activation rather than osteoblast-mediated bone formation in osteoporosis finally leads to loss of bone mass. ${ }^{18}$ The clinically osteoporotic patient has significant decreased levels of BMD and BMC. BMD is well known as a hallmark of diagnosis for bone structural deterioration. In addition to BMD, BMC is a one of the primary standards to analyze mechanical strength. ${ }^{19}$ The recovery of bone parameters, BMD and BMC, is strongly associated with loss of bone. ${ }^{20}$

In this study, OVX-induced osteoporotic mice showed decreases of femur BMC and BMD compared to Sham group. Ca treatment significantly increased the both of BMC and BMD.
Similar to result from Ca group, there was significant increment of BMC level in $100 \mathrm{mg} \mathrm{kg}^{-1}$ Osteo-F-treated femur. The decreased BMD was markedly recovered in OF10 and OF100 groups (Fig. 3). In addition, the hyperplasia of growth plate was appeared in osteoporosis-like bone in OVX mice. Administration of Ca suppressed the growth plate thickness compared to OVX group. Increases of growth plate thickness were ameliorated by Osteo-F administration, indicating that Osteo-F attenuated hyperplasia of growth plate (Fig. 2). Furthermore, calcium concentration in serum as bone turnover marker was significantly decreased by OVX surgery. However, administration of 1 , 10 and $100 \mathrm{mg} \mathrm{kg}^{-1}$ Osteo-F markedly increased the calcium levels in a dose-dependent manner. Especially, the serum of calcium level was nearly recovered to normal range in $100 \mathrm{mg}$ $\mathrm{kg}^{-1}$ Osteo-F-treated group (Fig. 4), while the level of magnesium was not changed by Osteo-F (data not shown). These results suggest that Osteo-F, newly developed formula, is effective on bone formation by increase of serum calcium levels and has anti-osteoporotic effect in OVX mice.

To clarify the mechanisms of Osteo-F on osteoporosis, osteoblast differentiation-related factors such as Runx 2 and

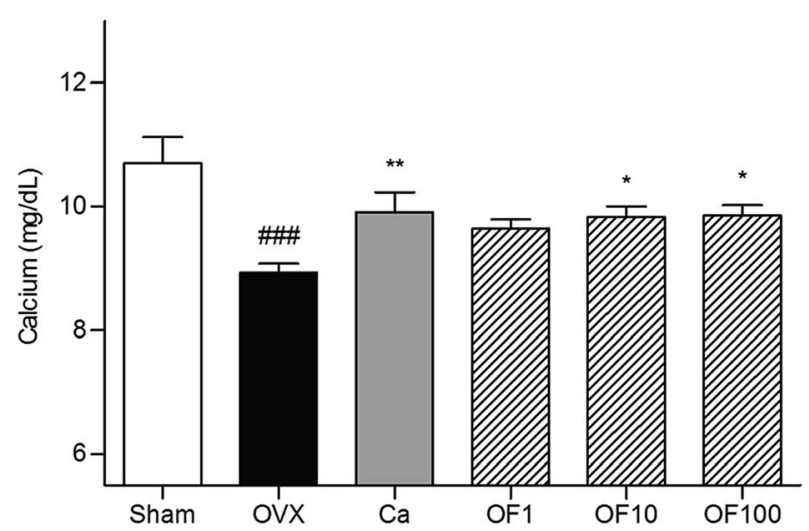

Fig. 4 The effects of Osteo-F on serum calcium concentrations. Results are presented as mean \pm S.D. \#\#\# indicate that the mean differs significantly between the Sham group and the OVX group ( $p<$ $0.001)$. and $* *$ indicate that the mean differs significantly between the OF or Ca group and the OVX group $(p<0.05$ and $p<0.01$, respectively)
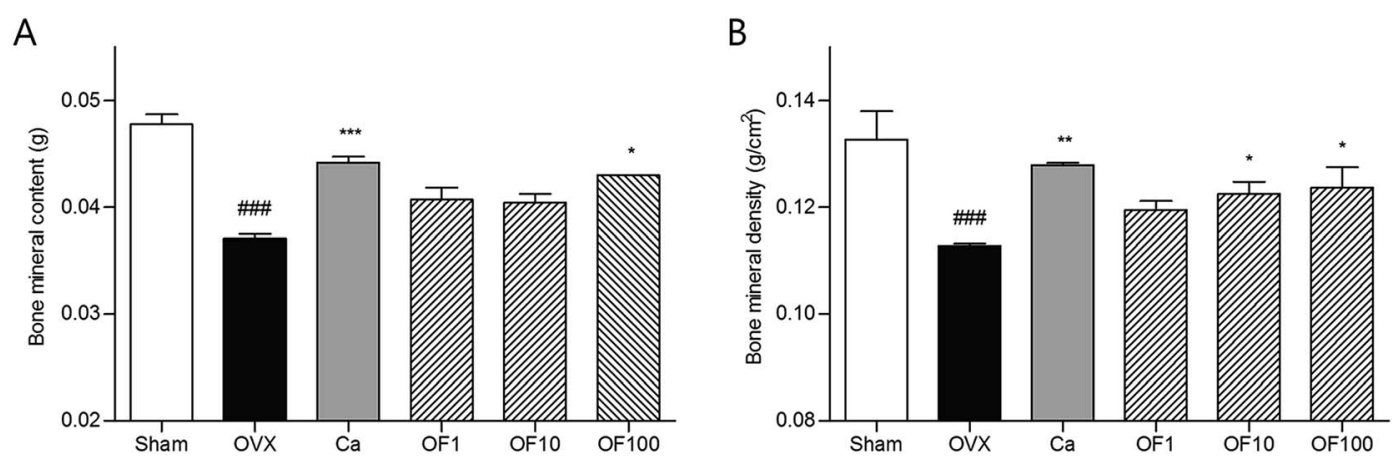

Fig. 3 The effects of Osteo-F on bone mineral content (BMC) and bone mineral density (BMD). Results are presented as mean \pm S.D. \#\#\# indicate that the mean differs significantly between the Sham group and the OVX group $(p<0.001)$. *,**and *** indicate that the mean differs significantly between the OF or Ca group and the OVX group ( $p<0.05, p<0.01$ and $p<0.001$, respectively). 

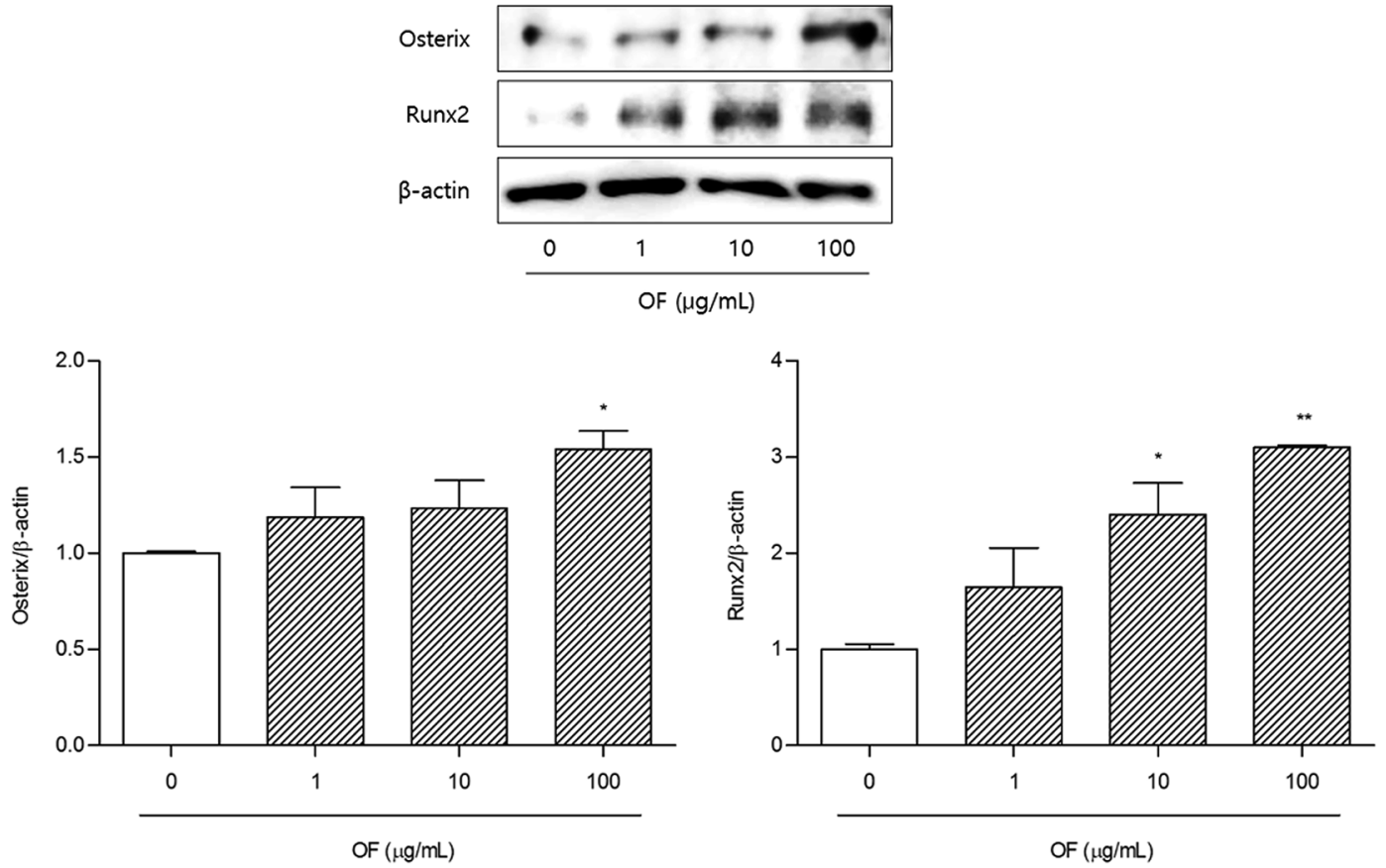

Fig. 5 The effects of Osteo-F on Runx2 and Osterix expressions in SaOS-2 cells. Data are represented as the means \pm S.D. of three independent experiments. * and ** indicate that the mean differs significantly between the OF-treated cell and the non-treated cells $(p<0.05$ and $p<0.01$, respectively).

Osterix were determined in osteoblast like SaOS-2 cells. Two transcriptional factors, Runx2 and Osterix, have been reported to be essential for osteoblast differentiation and maturation from pre-osteoblasts. ${ }^{21}$ Because bone formation depends on differentiation of osteoblast (bone-forming cells), the expressions of Runx2 and Osterix are regarded as osteogenic transcriptional factors for skeletal development. ${ }^{22,23}$ The expression level of Runx2 protein was significantly increased by $100 \mu \mathrm{g}$ $\mathrm{mL}^{-1}$ Osteo-F treatment in SaOS-2 osteoblast cells. Similarly, 10 and $100 \mu \mathrm{g} \mathrm{mL}^{-1}$ Osteo-F treatment up-regulated the expression of Osterix protein, compared to non-treated cells (Fig. 5). Especially, $100 \mu \mathrm{g} \mathrm{mL}^{-1}$ Osteo-F treatment significantly increased the both of Osterix and Runx2. All concentrations of Osteo-F exerted equivalent effects on SaOS-2 cell viability. OsteoF was not cytotoxic to SaOS-2 cell (data not shown). Osteo-F could differentiate from pre-osteoblasts to mature osteoblasts by up-regulating Runx2 and Osterix demonstrating the potential mechanism of action of Osteo-F on bone remodeling, especially osteoblast differentiation.

\section{Conclusions}

Osteo-F, a newly combined formula, has anti-osteoporotic effects in OVX mice. The levels of BMC and BMD were ameliorated, and histological changes of femur were recovered by Osteo-F treatment. In addition, Osteo-F showed bone remodeling by promoting osteoblast differentiation via up-regulation of Runx2 and Osterix. Taken together, Osteo-F could be one of the alternative materials for the improvement of osteoporosis.

\section{Conflict of interest}

The authors declare no conflict of interest.

\section{Acknowledgements}

This work was carried out with the support of "Cooperative Research Program for Agriculture Science \& Technology Development (PJ011668)" Rural Development Administration, Republic of Korea.

\section{References}

1 T. C. Phan, J. Xu and M. H. Zheng, Histol. Histopathol., 2004, 19, 1325-1344.

2 S. R. Cummings and L. J. Melton, Lancet, 2002, 359, 17611767.

3 N. Harvey, E. Dennison and C. Cooper, Nat. Rev. Rheumatol., 2010, 6, 99-105.

4 B. L. Clarke and S. Khosla, Radiol. Clin., 2010, 48, 483-495.

5 J. A. Cauley, Steroids, 2015, 99, 11-15.

6 K. Venken, F. Callewaert, S. Boonen and D. Vanderschueren, Osteoporosis Int., 2008, 19, 1517-1525.

7 J. E. Rossouw, G. L. Anderson, R. L. Prentice, A. Z. LaCroix, C. Kooperberg, M. L. Stefanick, R. D. Jackson, S. A. Beresford, B. V. Howard, K. C. Johnson, J. M. Kotchen and J. Ockene, J. Am. Med. Assoc., 2002, 288, 321-333.

8 T. J. Bunch, J. L. Anderson, H. T. May, J. B. Muhlestein, B. D. Horne, B. G. Crandall, J. P. Weiss, D. L. Lappe, J. S. Osborn and J. D. Day, Am. J. Cardiol., 2009, 103, 824-828. 
9 N. Yarom, R. Yahalom, Y. Shoshani, W. Hamed, E. Regev and S. Elad, Osteoporosis Int., 2007, 18, 1363-1370.

$10 \mathrm{~J}$. Heo, DONGUIBOGAM Treasured Mirror of Eastern Medicine, BUBIN PUBLISHERS CO, Seoul, Republic of Korea, fourth edn, 2005, pp. 765-766.

11 M. H. Kim, Y. Y. Choi, J. M. Han, H. S. Lee, S. B. Hong, S. G. Lee and W. M. Yang, Food Funct., 2014, 5, 1594-1601.

12 J. Yin, Y. Tezuka, K. Kouda, Q. L. Tran, T. Miyahara, Y. Chen and S. Kadota, Biol. Pharm. Bull., 2004, 27, 583-586.

13 J. E. Lee, M. H. Kim, Y. Y. Choi, H. J. Lee and W. M. Yang, Orient. Pharm. Exp. Med., 2016, 16, 53-57.

14 T. Luhmann, O. Germershaus, J. Groll and L. Meinel, J. Controlled Release, 2012, 161, 198-213.

15 M. Mittal and N. Chattopadyay, Indian J. Endocrinol. Metab., 2012, 16, S279-S281.
16 W. Sipos, P. Pietschmann, M. Rauner, K. Kerschan-Schindl and J. Patsch, Wien. Med. Wochenschr., 2009, 159, 230-234.

17 M. L. Brandi, Rheumatology, 2009, 48(suppl. 4), 3-8.

18 G. Karsenty, Genes Dev., 1999, 13, 3037-3051.

19 S. Satpathy, A. Patra and B. Ahirwar, J. Complementary Integr. Med., 2015, 12, 251-266.

20 M. Kleerekoper, Osteoporosis Int., 2006, 17, 1707-1715.

21 T. Komori, J. Cell. Biochem., 2006, 99, 1233-1239.

22 N. Nadiminty, W. Lou, S. O. Lee, F. Mehraein-Ghomi, J. S. Kirk, J. M. Conroy, H. Zhang and A. C. Gao, Clin. Cancer Res., 2006, 12, 1420-1430.

23 X. Zhou, Z. Zhang, J. Q. Feng, V. M. Dusevich, K. Sinha, H. Zhang, B. G. Darnay and B. de Crombrugghe, Proc. Natl. Acad. Sci. U. S. A., 2010, 107, 12919-12924. 